\title{
Anomeric Selectivity of Trehalose Transferase with Rare L-Sugars
}

\author{
Luuk Mestrom, Stefan R. Marsden, Hessel van der Eijk, Jesper U. Laustsen, Cy M. Jeffries, \\ Dmitri I. Svergun, Peter-Leon Hagedoorn, Isabel Bento,* and Ulf Hanefeld*
}

Cite This: ACS Catal. 2020, 10, 8835-8839

Read Online

\section{ACCESS | Llll Metrics \& More | 回 Article Recommendations | sl Supporting Information}

ABSTRACT: Retaining LeLoir glycosyltransferases catalyze the formation of glycosidic bonds between nucleotide sugar donors and carbohydrate acceptors. The anomeric selectivity of trehalose transferase from Thermoproteus uzoniensis was investigated for both D- and L-glycopyranose acceptors. The enzyme couples a wide range of carbohydrates, yielding trehalose analogues with conversion and enantioselectivity of $>98 \%$. The anomeric selectivity inverts from $\alpha, \alpha-(1 \rightarrow 1)$-glycosidic bonds for Dglycopyranose acceptors to $\alpha, \beta-(1 \rightarrow 1)$-glycosidic bonds for $\mathrm{L}$ glycopyranose acceptors, while $(S)$-selectivity was retained for both types of sugar acceptors. Comparison of protein crystal structures of trehalose transferase in complex with $\alpha, \alpha$-trehalose and an

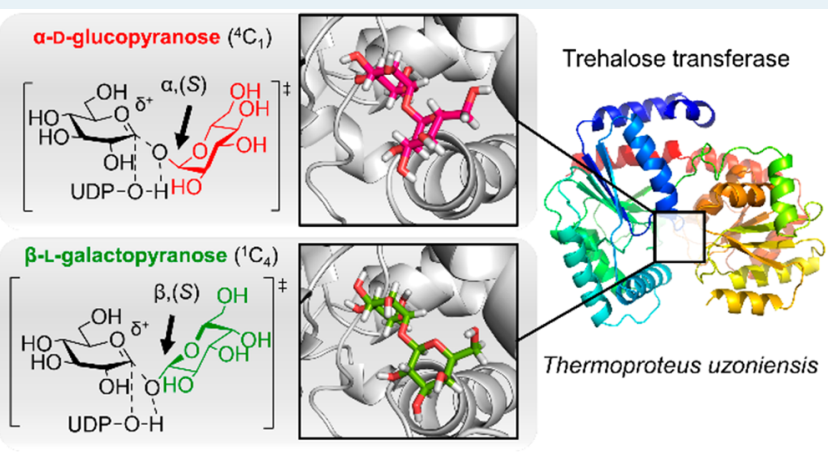
unnatural $\alpha, \beta$-trehalose analogue highlighted the mechanistic rationale for the observed inversion of anomeric selectivity.

KEYWORDS: glycosyltransferase, glycosidic linkages, trehalose, transferase, Thermoproteus uzoniensis

$\mathrm{T}$ he synthesis of a glycosidic bond is one of the most important reactions within glycochemistry. Enzymes couple sugars to afford oligosaccharides with high selectivity under mild reaction conditions. As enzymes are regarded to have evolved toward the selective conversion of the naturally more abundant D-sugars, their L-sugar enantiomers are often not considered as suitable substrates for enzymatic conversions. For this reason, the coupling of both $\mathrm{D}$ - and Lglycopyranose acceptors has rarely been compared for a single enzyme. However, the incorporation of L-sugars offers a broad spectrum of diametrically opposed glycosides or oligosaccharides, which might display new biological activities.

In one example, a retaining non-LeLoir glycosyltransferase (GT) coupled L-glycopyranose acceptors with sucrose as sugar donor in an $\alpha, \beta$ - $(1 \rightarrow 2)$-fashion, while $\alpha, \alpha$-( $1 \rightarrow 2)$-glycosidic bonds were observed with D-glycopyranose acceptors. ${ }^{1}$ The switch of anomeric selectivity for the sugar acceptor was attributed to the ${ }^{4} \mathrm{C}_{1}$ and ${ }^{1} \mathrm{C}_{4}$ chair configuration for $\mathrm{D}$ - and Lglycopyranoses, which affect the position of the nucleophilic hydroxyl group at the anomeric position. In general, $\alpha$-D and $\beta$ $\mathrm{L}$ anomers of the same sugar are structurally more alike (Figure 1a), than the corresponding $\alpha$-D and $\alpha$-L anomers (Figure 1b). ${ }^{2}$ This structural similarity allows the conversion of both $\alpha$-D and $\beta$-L configured substrates by an $(S)$-selective enzyme.

For retaining glycosyltransferases (GTs) with an internal nucleophilic substitution $\left(\mathrm{S}_{\mathrm{N}} \mathrm{i}\right)$ mechanism, the anomeric selectivity can be expected to invert when $(S)$-selectivity is retained (Figure 1c,d). ${ }^{3-5}$ The "same-face" attack of the nucleophile (i.e., sugar acceptor) on the leaving group (i.e., sugar donor) is guided by hydrogen bonding and proceeds with high anomeric selectivity for the sugar donor and acceptor. ${ }^{4}$ The position of the anomeric hydroxyl of the sugar acceptor might affect the type of glycosidic bond formed.

Here, the anomeric selectivity of the retaining LeLoir GT trehalose transferase (TreT) was investigated. TreT is particularly suitable for the screening of L-glycopyranoses, as it couples nucleotide diphosphate (NDP) sugar donors to a wide spectrum of nonphosphorylated D-sugar acceptors, resulting in an $\alpha, \alpha-(1 \rightarrow 1)$-glycosidic linkage. ${ }^{5}$ We focused on the recently described TreT from Thermoproteus uzoniensis ( $T u T r e T)$ fused to mCherry for the systematic screening of Dand L-glycopyranoses as sugar acceptors. ${ }^{6,7}$ mCherry TuTreT is an interesting enzyme because of a high thermostability, high activity, the possibility of fluorometric detection that is due to mCherry, and performance as an immobilized catalyst. ${ }^{8}$

Initially, the reaction conditions were optimized to exclude any possible side reactions or promiscuous activities. TuTreT did not display any phosphorylase or hydrolase activity. The use of glucose-1-phosphate as sugar donor did not result in the formation of trehalose (excluding phosphorylase activity), and

Received: May 12, 2020

Revised: July 21, 2020

Published: July 22, 2020 

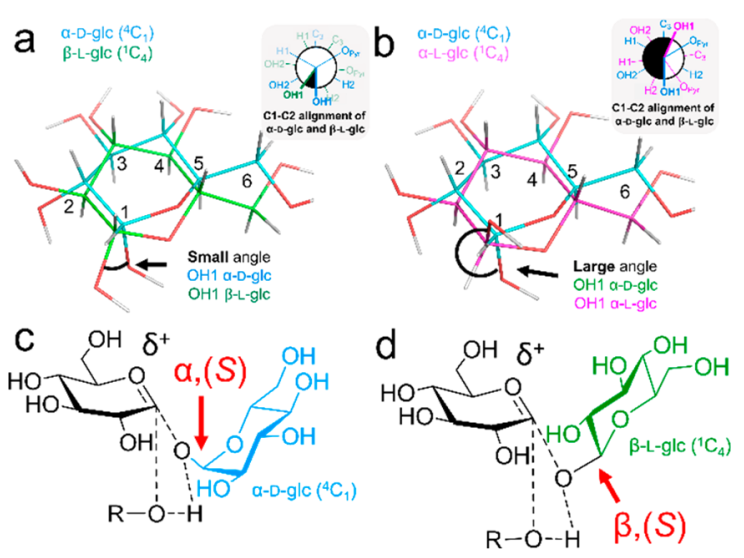

Figure 1. Position of the anomeric hydroxyl of $\alpha$-D-glucopyranose $\left({ }^{4} \mathrm{C}_{1}\right.$, cyan $)$ when superimposed to $\beta$-L-glucopyranose $\left({ }^{1} \mathrm{C}_{4}\right.$, green $)$ in panel a is more similar than $\alpha$-L-glucopyranose $\left({ }^{1} \mathrm{C}_{4}\right.$, purple) in panel b. The insets in panel a, b show the representative overlay of the Newman projection of the anomeric $\mathrm{OH} 1$. The $\mathrm{S}_{\mathrm{N}} \mathrm{i}$-like reaction mechanism with an oxocarbenium transition state $\left({ }^{4} \mathrm{H}_{3}\right)$ that allows the approach of the anomeric hydroxyl, $\alpha-\mathrm{OH}$ of D-glucose $\left({ }^{4} \mathrm{C}_{1}\right)$ in panel c, or the $\beta-\mathrm{OH}$ of $\mathrm{L}$-glucose $\left({ }^{1} \mathrm{C}_{4}\right)$ in panel $\mathrm{d}$, is guided by hydrogen bonding from the same face as the NDP leaving group. $\mathrm{R}=$ NDP.

no hydrolase activity was observed when the enzyme was incubated solely with trehalose. However, slow hydrolysis of uridine diphosphate-glucose (UDP-glucose) to UDP and glucose by TuTreT was observed, resulting in the subsequent formation of trehalose from glucose and UDP-glucose (Figure S3). To minimize the undesired formation of trehalose as a side product via UDP glucose hydrolysis during the screening of other sugar acceptors, the reaction time was limited to 60 min using $1.0 \mathrm{mg} \mathrm{mL}^{-1}$ of TuTreT.,10

Using these optimized conditions, the substrate tolerance of TuTreT was probed in a HPLC-based screening of D- and Lsugars (Figure 2). Conversion of L-glycopyranoses resulted in the hypothesized $\beta$-selectivity for TuTreT. Successful enzymatic conversions were repeated on preparative scale and the obtained trehalose analogues were analyzed by NMR and HRMS (Supporting Information). D-Glucose, D-mannose, Dgalactose, and D-xylose exclusively led to the formation of $\alpha, \alpha$ - $(1 \rightarrow 1)$-linked trehalose derivatives, while L-glucose, Lgalactose, and L-gulose led to the formation of $\alpha, \beta-(1 \rightarrow 1)$ linked trehalose derivatives. The long-range $\mathrm{C}-\mathrm{H}$ coupling over the glycosidic linkage confirmed the direct coupling of the $\mathrm{Cl}_{\text {acceptor }}$ with the $\mathrm{H1}^{\prime}{ }_{\text {donor }}$ and vice versa in $\mathrm{gHMBC}$ experiments. The ${ }^{4} \mathrm{C}_{1}$ configuration of $\alpha$-D- $\alpha$-D-glycopyranosides was confirmed by $J_{1,2}$ coupling $(\sim 4 \mathrm{~Hz})$ of the anomeric protons, which are gauche configured. The anomeric protons of $\beta$-L-glycopyranosides with a ${ }^{1} \mathrm{C}_{4}$ chair conformation are anti configured, resulting in larger $J_{1,2}$ coupling constants $(\sim 8 \mathrm{~Hz}){ }^{1}$

Further analysis of the HPLC screening demonstrates that Dand L-enantiomers of glucose and galactose were accepted, but L-mannose was not. For L-gulose, L-allose, and L-altrose, their D-glycopyranoses enantiomers were not accepted. Anomeric selectivity is dictated by more than the anomeric configuration, and the overall conformation of the sugar acceptor is important as well. The structural variants of D-glycopyranoses with a ${ }^{4} C_{1}$ configuration were readily converted, such as D-glucose, $\mathrm{D}$ mannose, and D-galactose. This is in line with results for other TreTs. $^{11,12}$ C5 sugars were generally less well accepted, with

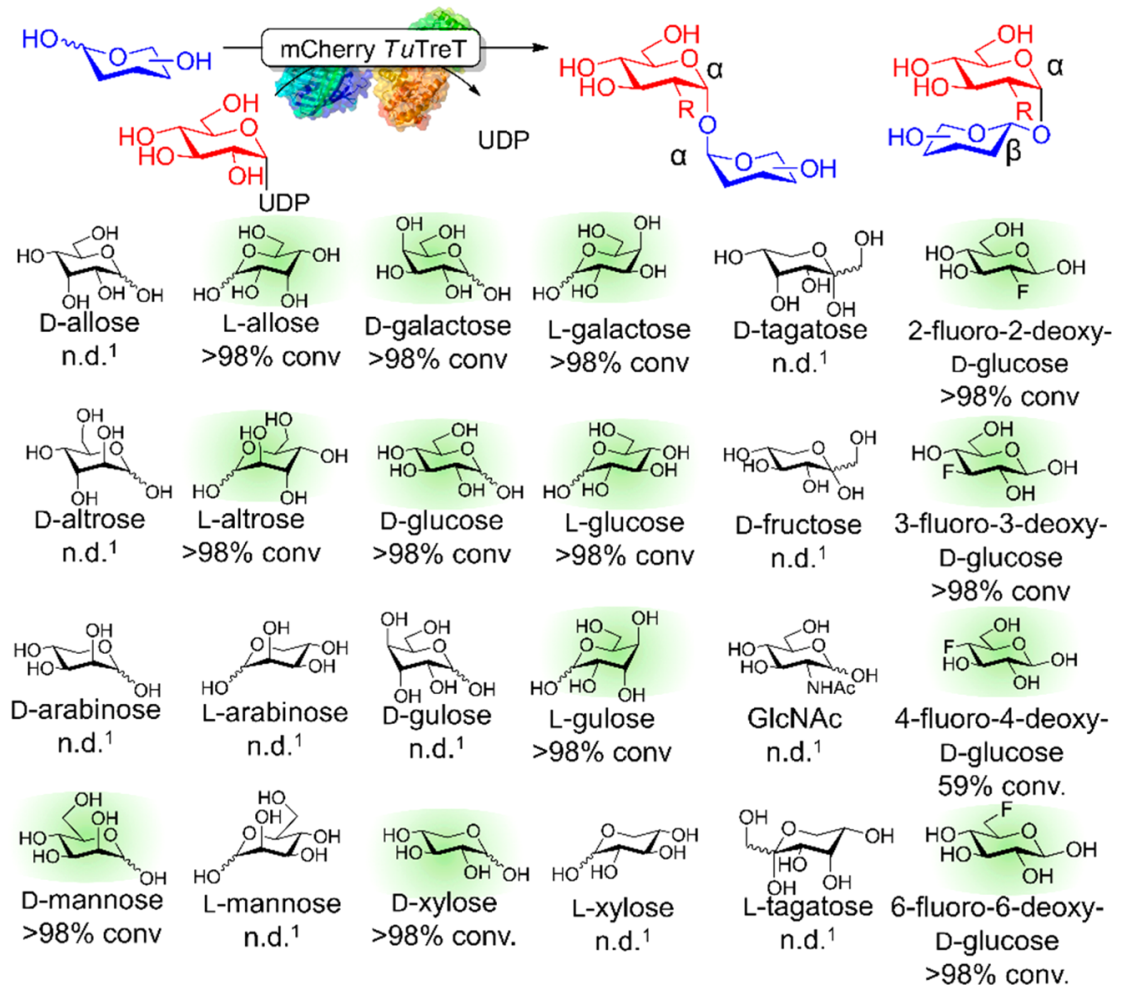

Figure 2. mCherry TuTreT catalyzed conversion of D- and L-sugar acceptors with UDP-D-glucose as donor for the screening for the formation of product. The conversion was determined using external calibration curves of sugar acceptor with HPLC. ${ }^{1}$ n.d: not detected including a trehalose analogue product. Reaction conditions: substrate (10 mM), UDP-D-glucose (40 mM), HEPES (50 mM), $\mathrm{MgCl}_{2}(20 \mathrm{mM})$, mCherry TuTreT (13.5 $\mathrm{nM}), \mathrm{pH} 7.0,60{ }^{\circ} \mathrm{C}, 1 \mathrm{~h}$ of reaction time. 

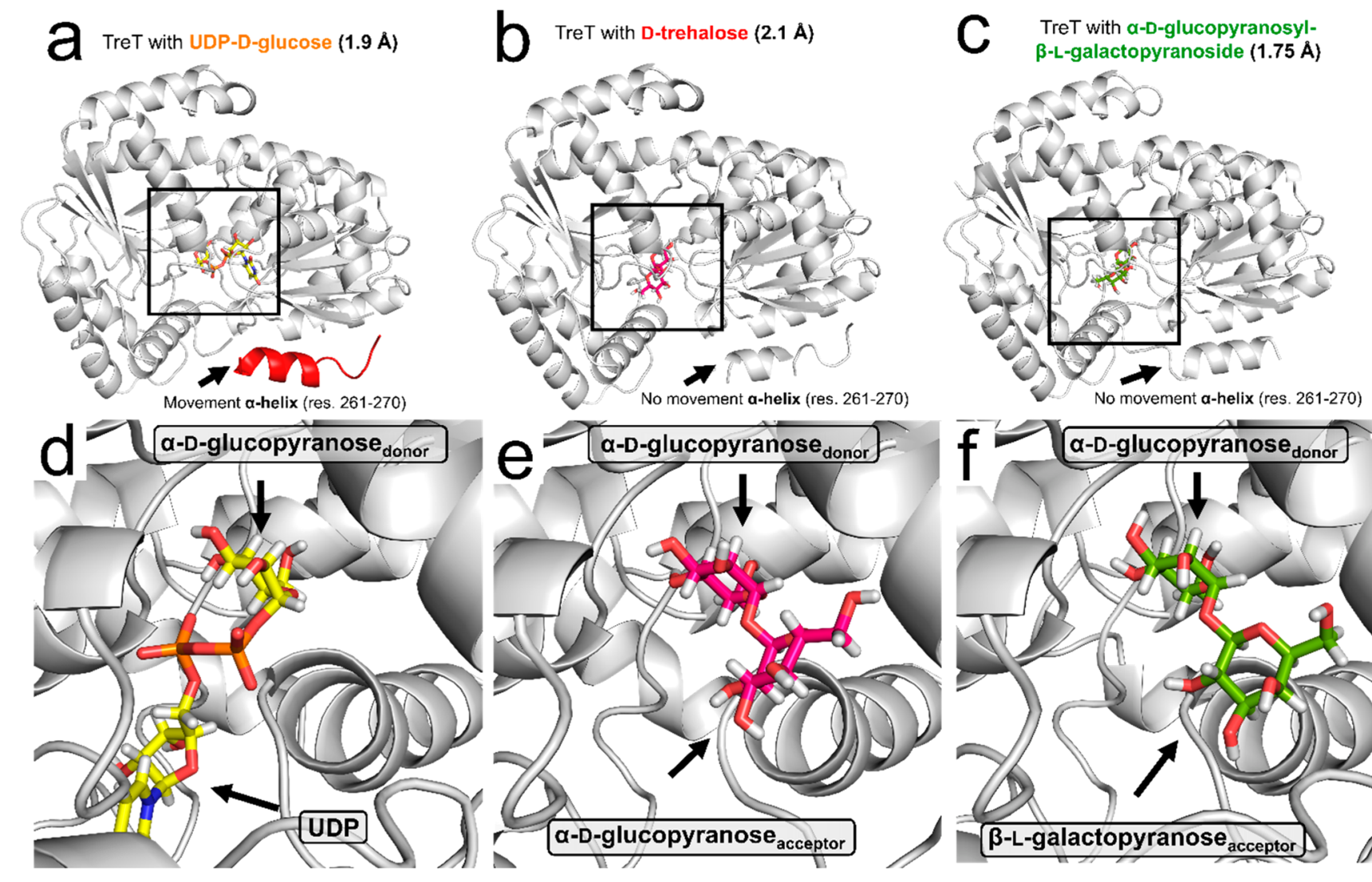

Figure 3. Protein crystal structure of TuTreT containing UDP-D-glucose in panel a (PDB: 6ZMZ, inset in panel d), TuTreT bound with $\alpha, \alpha$ trehalose in panel b (PDB: 6ZJH, inset shown in panel e), and TuTreT in complex with enzymatically synthesized $\alpha$-D-glucopyranosyl- $\beta$-Lgalactopyranose in panel c (PDB: 6ZN1, inset shown in panel f). The nature of the glycosidic bond is demonstrated showing a high overlap for the sugar donor and the glycosidic linkages between panels $\mathrm{d}$ and $\mathrm{e}$, or panels $\mathrm{d}$ and $\mathrm{f}$, while the orientation of the sugar acceptor changes slightly between panels e and $\mathrm{f}$.

the exception of D-xylose, which lacks a $\mathrm{CH}_{2} \mathrm{OH}$ group in comparison to D-glucose. The ketohexopyranoses D-fructose and $\mathrm{D}$ - and L-tagatose were not converted under the conditions provided, which display dissimilar overall structural conformation of the cyclic ring structure as well as the anomeric configuration. Interactions between active site residues and the carbohydrate substrate were investigated by extending the substrate screening to fluorodeoxy-carbohydrates. Unlike hydroxyl groups, fluorine can exclusively function as a hydrogen bond acceptor. All fluoro-deoxy-D-glucopyranoses were quantitatively converted as acceptor substrates, with the exception of 4-deoxy-4-fluoro-D-glucose. ${ }^{11}$ The interaction of hydrogen bond donor 4-OH of the sugar acceptor with the deprotonated Asp254 of TuTreT is possibly important for acceptor substrate recognition.

The substrate tolerance toward the glycopyranose moiety of the sugar donor and the sugar acceptor is distinct from one another. ${ }^{13}$ For instance, the coupling of UDP-D-glucose with $\mathrm{N}$-acetyl-D-glucosamine (GlcNAc) did not result in any observable conversion. The sugar donor UDP-D-GlcNAc and D-glucose is readily converted by TuTreT with $>98 \%$ conversion and $\alpha, \alpha-(1 \rightarrow 1)$-selectivity.

As this is the first report that the $\alpha$-D-selective retaining glycosyltransferase TreT catalyzes the glycosidic bond formation with $\beta$-L-glycopyranose acceptors, the protein crystal structure of TuTreT was studied. As the mCherry TuTreT fusion construct did not crystallize satisfactorily, the glycinerich linker of the fusion protein was cleaved using "in situ" proteolysis with retention of enzyme activity (Figure S1), and the protein was purified (Figure S2). The protein without the mCherry tag subsequently crystallized as apo (PDB: 6ZJ4, 2.1 Å resolution), cocrystallized with magnesium(II) (PDB: 6ZJ7, $2.15 \AA$ resolution), or soaked with D-trehalose (PDB: 6ZJH, $2.1 \AA$ resolution), D-glucopyranosyl-L-galactopyranose (PDB: $6 \mathrm{ZN1} 1,1.75 \AA$ resolution), and UDP- $\alpha$-D-glucose (PDB: 6ZMZ, 1.9 ̊ resolution). The latter three are shown in Figure $3 a-c$.

The overall three-dimensional fold observed in all determined crystal structures are similar to the one found in trehalose phosphate synthase (OtsA, PDB: 1GZ5, RMSD of $2.0 \AA$ for $304 \mathrm{C} \alpha)^{14}$ from $E$. coli and in trehalose transferase from Pyrococcus horikoshii (PDB: 2X6Q, RMSD of $2.5 \AA$ for $363 \mathrm{C} \alpha),{ }^{15}$ showing in each domain a characteristic Rossmann fold (Figure S4). Furthermore, all TuTreT structures show a monomer in the asymmetric unit, and this state was confirmed by size-exclusion small-angle X-ray scattering (SEC-SAXS) measurements in aqueous solution (Figure S9-S10). ${ }^{16} \mathrm{We}$ conclude that the functional unit of TuTreT is a monomer, whereas a dimer has been described for PhTreT $^{15}$ and a tetramer for OtsA from E. coli. ${ }^{14}$ The overall protein conformation remained unchanged when bound to ligands in aqua, according to SEC-SAXS (Figure S9-S10). The protein crystal structures also show high structural similarity (Table S3); however, a minor conformational change was observed for the sugar donor binding site of TuTreT when it was soaked with UDP-D-glucose. Hydrogen bond interactions between the 
uracil moiety and a disordered loop region of the protein (Figure S6, res. 250-262), were inducing a shift of an $\alpha$-helix by $2.0 \AA$ (Figure S5). This finding is not in agreement for what has been found for PhTreT, where a larger conformational change was observed for the whole domain after soaking the crystals with trehalose. ${ }^{15}$

The active site of TuTreT is located between the $\mathrm{N}$ - or $\mathrm{C}$ terminal domains of the acceptor and donor binding sites (Figure S4). Substrate-bound structures demonstrated clear electron densities at the active site (Figure S6). The active site residues for the sugar donor and acceptor binding sites are conserved for TuTreT, PhTreT, ${ }^{15}$ and OtsA (Figure S7). ${ }^{14}$ In TuTreT, the pyrophosphate moiety of the nucleotide sugar donor interacts with Arg221 and Lys226, and the active site can accommodate pyrimidine or purine nucleobases (Figure 3a,d). This leads to the ability of the enzyme to convert nucleotide sugar donors with different nucleotides, which holds for TreTs in general, ${ }^{17,18}$ as was observed with UDP- and ADP-D-glucose with TuTreT previously. ${ }^{6,7}$

The natural product $\alpha, \alpha$-D-trehalose shows an $\alpha, \alpha-(1 \rightarrow 1)$ glycosidic bond when bound to TuTreT (Figure $3 \mathrm{~b}, \mathrm{e}$ ). In trehalose, the $\alpha$-D-glucopyranose donor $_{\text {moiety binds at the same }}$ sugar binding site as the UDP- $\alpha$-D-glucopyranose donor (Figure $\mathrm{S} 8 \mathrm{a}-\mathrm{c}$ ). The sugar donor binding site of UDP-Dglucose of TuTreT (shown in Figure S8d) is similar to what has been reported for TreT from Pyrococcus horikishii. ${ }^{15}$ Interestingly, the $\alpha, \alpha$ - and $\alpha, \beta$-trehalose derivatives the $\alpha$-D-

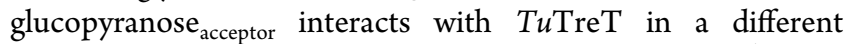
binding mode than the $\beta$-L-galactopyranose acceptor $_{\text {(Figure }}$ $\mathrm{S} 8 \mathrm{e}, \mathrm{f})$. Arg221 might bind with the $2 \mathrm{OH}$ and $3 \mathrm{OH}$ of the $\beta$ L-galactopyranose acceptor (Figure S8e), while the $3 \mathrm{OH}$ and $4 \mathrm{OH}$ of $\alpha$-D-glucopyranose $e_{\text {acceptor }}$ is at closer distance to $\operatorname{Arg} 221$ (Figure S8f). The movement of the highly conserved Arg221 when UDP-D-glucose is bound was notable (Figure S5f), which has been postulated to play a role in substrate recognition in PhTreT. ${ }^{15}$ Also, Asp256 is at a hydrogen bonding distance to $4 \mathrm{OH}$ of $\alpha$-D-galactopyranose acceptor $_{\text {(Figure S8e), while no clear }}$ electron density for this loop could be found for the natural $\alpha$ D-glucopyranose acceptor The $\alpha$-D-glucopyranose acceptor $_{\text {moiety of }}$ trehalose is directed into the wide cavity of TuTreT.

Within the protein crystal structure, the conformation of the carbohydrates demonstrated for the enzymatically synthesized $\alpha, \beta-(1 \rightarrow 1)$-L-galactotrehalose (Figure $3 \mathrm{c}, \mathrm{f})$, a ${ }^{1} \mathrm{C}_{4}$ conformation of the $\beta$-L-galactopyranose moiety. As was shown in Figure 1, the anomeric $\beta-\mathrm{OH}_{\text {acceptor }}$ hydroxyl group points into the same direction as the $\alpha-\mathrm{OH}_{\text {acceptor }}$ hydroxyl group in the natural substrate D-trehalose (Figure $3 \mathrm{f}$ ). Hence, the overall geometry of $\alpha$-D-glucopyranose with a ${ }^{4} \mathrm{C}_{1}$ conformation and $\beta$-L-galactopyranose with a ${ }^{1} \mathrm{C}_{4}$ conformation are highly similar (Figure S8g,h).

On the basis of these findings, we hypothesized that highly (S)-selective TuTreT guides the anomeric hydroxyl of the sugar acceptor according to a $\mathrm{S}_{\mathrm{N}} \mathrm{i}$-like mechanism. More specifically, the $\alpha-\mathrm{OH}$ of D-glucose $\left({ }^{4} \mathrm{C}_{1}\right)$, or the $\beta-\mathrm{OH}$ of $\mathrm{L}$ galactose $\left({ }^{1} \mathrm{C}_{4}\right)$ are guided by hydrogen bonding from the same face as the NDP leaving group. As the $S_{N}$ i-like mechanism requires the same-face participation with the anomeric hydroxyl of the nucleotide phosphate of the glycosyl donor, the sugar coupling does not readily proceed for the equatorial $\beta-\mathrm{OH}$ of D-glucose $\left({ }^{4} \mathrm{C}_{1}\right)$ or the axial $\alpha-\mathrm{OH}$ of Lgalactose $\left({ }^{1} \mathrm{C}_{4}\right)$. This allows $\mathrm{TuTreT}$ to retain its $(S)$-selectivity for the anomeric hydroxyl group, while the anomeric configuration inverts. This mechanistic rationale explains the inversion of anomeric selectivity of TuTreT with L-glycopyranose acceptors, emphasizing that understanding the structural conformations of hexopyranoses is important for understanding enzyme selectivity when glycosidic bonds are formed. This inversion of anomeric selectivity might not be limited to TuTreT and could occur in other GTs as well.

In conclusion, mCherry TuTreT catalyzes the formation of trehalose derivatives with a large substrate spectrum. The switch in anomeric selectivity for D- and L-sugar acceptors can be explained on the basis of structural conformations of carbohydrates, leading to the formation of distinctive $\alpha$-D- $\alpha$-Dor $\alpha$-D- $\beta$-L-glycosidic linkages. This paves the way for further studies of utilizing rare L-glycopyranoses with retaining LeLoir glycosyltransferases, which are especially interesting for the production of oligosaccharides and glycans with unnatural glycosidic linkages.

\section{ASSOCIATED CONTENT}

\section{SI Supporting Information}

The Supporting Information is available free of charge at https://pubs.acs.org/doi/10.1021/acscatal.0c02117.

Full experimental detail including fermentation, production and purification of the enzyme, details of the enzyme catalyzed conversions, HPLC and HRMS and NMR data for the complete characterization of the products, SAXS data and X-ray crystal data of the enzyme and DNA and protein sequence (PDF)

\section{AUTHOR INFORMATION}

\section{Corresponding Authors}

Ulf Hanefeld - Department of Biotechnology, Delft University of Technology, 2629 HZ Delft, The Netherlands; ㅇo이.org/ 0000-0002-4102-6165; Email: U.Hanefeld@tudelft.nl

Isabel Bento - European Molecular Biology Laboratory, EMBL, 22607 Hamburg, Germany; Email: Isabel.Bento@EMBLHamburg.de

\section{Authors}

Luuk Mestrom - Department of Biotechnology, Delft University of Technology, 2629 HZ Delft, The Netherlands

Stefan R. Marsden - Department of Biotechnology, Delft University of Technology, $2629 \mathrm{HZ}$ Delft, The Netherlands

Hessel van der Eijk - Department of Biotechnology, Delft University of Technology, $2629 \mathrm{HZ}$ Delft, The Netherlands

Jesper U. Laustsen - European Molecular Biology Laboratory, EMBL, 22607 Hamburg, Germany

Cy M. Jeffries - European Molecular Biology Laboratory, EMBL, 22607 Hamburg, Germany

Dmitri I. Svergun - European Molecular Biology Laboratory, EMBL, 22607 Hamburg, Germany

Peter-Leon Hagedoorn - Department of Biotechnology, Delft University of Technology, $2629 \mathrm{HZ}$ Delft, The Netherlands

Complete contact information is available at:

https://pubs.acs.org/10.1021/acscatal.0c02117

\section{Author Contributions}

The manuscript was written through contributions of all authors. All authors have given approval to the final version of the manuscript.

Notes

The authors declare no competing financial interest. 


\section{ACKNOWLEDGMENTS}

Marc Strampraad, Dirk Geerts, and Rob Kerste are kindly acknowledged for their involvement in the production of mCherry TuTreT in E. coli Top10 cells. Carol de Ram and Martin Pabst are kindly acknowledged for their great support in the HR-MS measurements. The SAXS measurements were performed at the EMBL-P12 beamline, Petra-3 Hamburg. We acknowledge the technical support given by the SPC facility at EMBL Hamburg. This research was supported by grant ERAIB-15-110 of the ERA-NET on Industrial Biotechnology. Portions of the research received support from the Horizon 2020 program of the European Union, iNEXT [653706; D.I.S], and iNEXT-Discovery [871037].

\section{REFERENCES}

(1) Seibel, J.; Moraru, R.; Götze, S.; Buchholz, K.; Na’amnieh, S.; Pawlowski, A.; Hecht, H.-J. Synthesis of sucroseanalogues and the mechanism of actionof Bacillus subtilis fructosyltransferase (levansucrase). Carbohydr. Res. 2006, 341, 2335-2349.

(2) Bubb, W. A. NMR Spectroscopy in the Study of Carbohydrates: Characterizing the Structural Complexity. Concepts Magn. Reson. 2003, 19A, 1-19.

(3) Lairson, L. L.; Henrissat, B.; Davies, G. J.; Withers, S. G. Glycosyltransferases: Structures, Functions, and Mechanisms. Annu. Rev. Biochem. 2008, 77, 521-555.

(4) Lee, S. S.; Hong, S. Y.; Errey, J. C.; Izumi, A.; Davies, G. J.; Davis, B. G. Mechanistic evidence for a front-side, $S_{\mathrm{N}} \mathrm{i}$-type reaction in a retaining glycosyltransferase. Nat. Chem. Biol. 2011, 7, 631-638.

(5) Ardèvol, A.; Rovira, C. The Molecular Mechanism of Enzymatic Glycosyl Transfer with Retention of Configuration: Evidence for a Short-Lived Oxocarbenium-Like Species. Angew. Chem., Int. Ed. 2011, 50, 10897-10901.

(6) Mestrom, L.; Marsden, S. R.; Dieters, M.; Achterberg, P.; Stolk, L.; Bento, I.; Hanefeld, U.; Hagedoorn, P.-L. Artificial Fusion of mCherry Enhances Trehalose TransferaseSolubility and Stability. Appl. Environ. Microbiol. 2019, 85, e03084-18.

(7) Correction of ref 6: Mestrom, L.; Marsden, S. R.; Dieters, M.; Achterberg, P.; Stolk, L.; Bento, I.; Hanefeld, U.; Hagedoorn, P.-L. Correction for Mestrom et al., "Artificial Fusion of mCherry Enhances Trehalose Transferase Solubility and Stability. Appl. Environ. Microbiol. 2019, 85, e00942-19.

(8) Mestrom, L.; Marsden, S. R.; McMillan, D. G. G.; Schoevaart, R.; Hagedoorn, P.-L.; Hanefeld, U. Comparison of Enzymes Immobilised on Immobeads and Inclusion Bodies: A Case Study of a Trehalose Transferase. Chem CatChem 2020, 12, 3249-3256.

(9) Mestrom, L.; Przypis, M.; Kowalczykiewicz, D.; Pollender, A.; Kumpf, A.; Marsden, S. R.; Bento, I.; Jarzębski, A. B.; Szymańska, K.; Chruściel, A.; Tischler, D.; Schoevaart, R.; Hanefeld, U.; Hagedoorn, P.-L. Leloir Glycosyltransferases in Applied Biocatalysis: A Multidisciplinary Approach. Int. J. Mol. Sci. 2019, 20, 5263.

(10) Marsden, S. R.; Mestrom, L.; McMillan, D. G. G.; Hanefeld, U. Thermodynamically and Kinetically Controlled Reactions in Biocatalysis - from Concepts to Perspectives. ChemCatChem 2020, 12, 426-437.

(11) Urbanek, B. L.; Wing, D. C.; Haislop, K. S.; Hamel, C. J.; Kalscheuer, R.; Woodruff, P. J.; Swarts, B. M. Chemoenzymatic Synthesis of Trehalose Analogues: Rapid Access to Chemical Probes for Investigating Mycobacteria. ChemBioChem 2014, 15, 2066-2070.

(12) Kim, H.-M.; Chang, Y.-K.; Ryu, S.-I.; Moon, S.-G.; Lee, S.-B. Enzymatic synthesis of a galactose-containing trehalose analogue disaccharide by Pyrococcus horikoshii trehalose-synthesizing glycosyltransferase: Inhibitory effects on several disaccharidase activities. J. Mol. Catal. B: Enzym. 2007, 49, 98-103.

(13) Groenevelt, J. M.; Meints, L. M.; Stothard, A. I.; Poston, A. W.; Fiolek, T. J.; Finocchietti, D. H.; Mulholand, V. M.; Woodruff, P. J.; Swarts, B. M. Chemoenzymatic Synthesis of Trehalosamine, an
Aminoglycoside Antibiotic and Precursor to Mycobacterial Imaging Probes. J. Org. Chem. 2018, 83, 8662-8667.

(14) Gibson, R. P.; Turkenburg, J. P.; Charnock, S. J.; Lloyd, R.; Davies, G. J. Insights into Trehalose Synthesis Provided by the Structure of the Retaining Glucosyltransferase OtsA. Chem. Biol. 2002, 9, 1337-1346.

(15) Woo, E.-J.; Ryu, S.-I.; Song, H.-N.; Jung, T.-Y.; Yeon, S.-M.; Lee, H.-A.; Park, B. C.; Park, K.-H.; Lee, S.-B. Structural Insights on the New Mechanism of Trehalose Synthesis by Trehalose Synthase TreT from Pyrococcus horikoshii. J. Mol. Biol. 2010, 404, 247-259.

(16) Blanchet, C. E.; Spilotros, A.; Schwemmer, F.; Graewert, M. A.; Kikhney, A.; Jeffries, C. M.; Franke, D.; Mark, D.; Zengerle, R.; Cipriani, F.; Fiedler, S.; Roessle, M.; Svergun, D. I. Versatile sample environments and automation for biological solution X-ray scattering experiments at the P12 beamline (PETRA III, DESY). J. Appl. Crystallogr. 2015, 48, 431-443.

(17) Ryu, S.-I.; Kim, J.-E.; Kim, E.-J.; Chung, S.-K.; Lee, S.-B. Catalytic reversibility of Pyrococcus horikoshii trehalose synthase: Efficient synthesis of several nucleoside diphosphate glucoses with enzyme recycling. Process Biochem. 2011, 46, 128-134.

(18) Qu, Q.; Lee, S.-J.; Boos, W. TreT, a Novel Trehalose Glycosyltransferring Synthase of the Hyperthermophilic Archaeon Thermococcus litoralis. J. Biol. Chem. 2004, 279, 47890-47897. 